\title{
EDITORIAL
}

\section{Artificial Intelligence and Pathobiology Join Forces in The American Journal of Pathology}

Stanley Cohen ${ }^{\star \dagger}$ and Martha B. Furie

From the Department of Pathology, * Perelman School of Medicine, University of Pennsylvania, Philadelphia, Pennsylvania; the Department of Pathology, ${ }^{\dagger}$ Sidney Kimmel Medical College, Philadelphia University and Thomas Jefferson University, Philadelphia, Pennsylvania; the Department of Pathology, ${ }^{\ddagger}$ Feinberg School of Medicine, Northwestern University, Chicago, Illinois; and the Department of Pathology, ${ }^{\S}$ Stony Brook University, Stony Brook, New York

Most new ventures are undertaken with a mixture of trepidation and enthusiasm, and certainly that was the case when the current Editor-in-Chief and Senior Associate Editors of The American Journal of Pathology (AJP) began their terms one year ago. Fortunately, the previous Editorin-Chief, Kevin Roth, gifted us with a strong foundation on which to build, including an uptick in the impact factor and a growing number of submissions. Consequently, 2018 has been a very positive year for $A J P$. AJP ranks first among 79 pathology journals in total number of citations and second in Eigenfactor Score, which is designed to measure the overall influence of a journal on its field (InCites Journal Citation Reports, https://jcr.incites.thomsonreuters.com, last accessed October 25, 2018).

Perhaps the most profound change that 2018 brought to $A J P$ was an explicit extension of its scope. Beginning in the 1980 s, priority was placed on mechanistic studies. However, today's investigations in pathology often generate large data sets that are descriptive but nonetheless inform future mechanistically-oriented inquiries. Such investigations are now specifically welcomed in AJP's statement of scope. Keeping AJP current with the changing nature of investigative pathology is essential to maintaining its relevancy and impact on the field. With that goal in mind, we are excited to inform AJP's readers of another expansion of scope. $A J P$ is now encouraging foundational, disease-related studies that incorporate deep learning and artificial intelligence. We consider this field of research to be an important evolution of our discipline.

The development of computer algorithms that learn from data and can extrapolate patterns and predictions (machine learning) has been the driving force toward a whole new paradigm for scientific research. The classic model is that data lead to explanatory mechanisms, which then suggest further experiments that lead to novel findings. This is the classic hypothesis-testing approach. Because of rapid technological progress, however, many experiments now collect vast amounts of information and can be considered hypothesisgenerating. Investigations in genomics and the other -omics are cases in point. The advent of image digitization also has led to experiments that generate gigabytes or terabytes of data. Fortunately, advances in a subset of machine learning known as deep learning or artificial intelligence allow us to derive important quantitative as well as qualitative information from images, thus putting visual observation on the same playing field as molecular analysis. Since deep learning is also very useful for -omic analysis, this approach enables the amalgamation and interpretation of image-based data with -omic information, thus allowing these data to be mined for new understanding. In this paradigm, we are hunting for meaning in a huge data set, instead of proceeding one logical step at a time from observation to explanation to new observations to better explanations, and so on in an iterative process. We have, in a certain manner of speaking, turned the scientific process on its end.

The unaided human brain finds it difficult, if not impossible, to extract meaning from such vast data sets. Thus, it is necessary to use computers to identify patterns and associations and make inferences and predictions from the data. A typical definition of machine learning is that it is a computational system based on a set of algorithms that attempt to model high-level abstractions in data by using multiple layers of analysis. There are a number of different ways in which a computer can be programmed to make intelligent judgments, and it is important for the investigator to use the right algorithm for a specific research project. The

Accepted for publication November 6, 2018.

Disclosures: None declared.

Address correspondence to Martha B. Furie, Ph.D., Room 248, Centers for Molecular Medicine, Stony Brook University, Stony Brook, NY 11794 5120. E-mail: martha.furie@stonybrookmedicine.edu. 
most powerful programs are based upon neural networks, which mimic the brain's wiring. But there are many others, including support vectors, Bayesian networks, K-nearest neighbors, and random forests. The subset of machine learning known as deep learning is what allows us to make optimal use of data embedded in images. We usually use the term artificial intelligence to include all of machine learning and not just deep learning. However, the term is misleading, as it implies computers can think and will someday replace us. As E. W. Dijkstra noted in a lecture given at the 1984 Association on Computing Machinery (ACM) Southern Central Regional Conference, "Asking if a computer can think is like asking if a submarine can swim." (https://Www. cs.utexas.edu/ EWD/transcriptions/EWD08xx/EWD898. html, last accessed October 25, 2018.) Currently, computer programs are not able to identify causal links among events; they can only expose correlations that suggest causality. We will be able to identify the (unlikely) moment in the future that this situation changes as the point in time when the computer rather than the pathologist gains first authorship and, ultimately, tenure.

To summarize, it is becoming increasingly necessary to use machine learning to expand the ability of our brains to make meaningful decisions based upon the information we obtain and the patterns we observe. Fortunately, we are seeing more and more collaborations among pathologists, engineers, computer scientists, and physicists. Unfortunately, computer scientists often find it hard to recognize what problems pathologists need to solve and what they are seeking in their data, and the pathologists may be unfamiliar with the strengths and limitations of the programs that the computer scientists generate. Consequently, papers published in computer journals frequently address trivial questions in pathology, whereas papers published in biomedical journals often use naïve algorithms. Happily, many students majoring in engineering and computer science are becoming drawn to careers in medicine, and they see pathology as a natural home for this integration of the sciences. This trend is important not only for the role of artificial intelligence in pathology but also for other computational aspects of our discipline. For example, we are seeing the rapid evolution of biophysical probes that generate spatial information beyond traditional optics. Examples include matrix-assisted laser desorption/ionization (MALDI), Raman imaging, and ultra-high resolution magnetic resonance imaging. These methods all require sophisticated computer processing for visualization and analysis and add to the complexity of scientific data acquisition and interpretation.

For all these reasons, it is likely that artificial intelligence will be as much of a game changer for pathology in the future as molecular biology has been over the past several decades. $A J P$ is dedicated to playing an enabling role in this evolution, as a place where good biological science and good computer science can meet in the service of the study of disease. To this end, AJP's statement of scope has been revised for 2019 and now reads:

The American Journal of Pathology, official journal of the American Society for Investigative Pathology and published by Elsevier, Inc., seeks high-quality original research reports, reviews, and commentaries related to the molecular and cellular basis of disease. The editors will consider basic, translational, and clinical investigations that directly address mechanisms of pathogenesis or provide a foundation for future mechanistic inquiries. Examples of such foundational investigations include data mining, identification of biomarkers, molecular pathology, and discovery research. Foundational studies that incorporate deep learning and artificial intelligence are also welcome. High priority is given to explorations of human disease and relevant experimental models using molecular, cellular, and organismal approaches.

Accordingly, we encourage submission of articles that use new, improved algorithms to advance the understanding of biomedical principles and pathogenesis. And we continue to welcome original research articles and review articles in all areas of investigative pathology. Full information for authors is available at https://ajp.amjpathol.org/content/ authorinfo, and questions can be sent to the Editorial Office at ajp@asip.org. 